\title{
ANÁLISE ESPECTROSCÓPICA DA MATÉRIA ORGÂNICA NO SEDIMENTO SUPERFICIAL DA BAÍA DE SEPETIBA, RIO DE JANEIRO, BRASIL
}

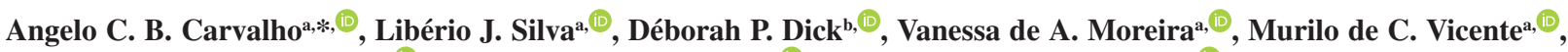

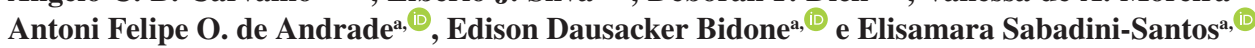 \\ ${ }^{a}$ Departamento de Geoquímica, Universidade Federal Fluminense, 24020-141 Niterói - RJ, Brasil \\ bDepartamento de Físico-Química, Instituto de Química, Universidade Federal do Rio Grande do Sul, 91501-970 Porto Alegre - \\ RS, Brasil
}

Recebido em 11/10/2019; aceito em 06/02/2020; publicado na web em 13/04/2020

\begin{abstract}
SPECTROSCOPIC ANALYSIS OF ORGANIC MATTER IN SURFACE SEDIMENTS FROM SEPETIBA BAY, RIO DE JANEIRO, BRAZIL. Spectroscopic techniques are powerful tools for molecular organic matter assessment in sediments, which analysis request low sample mass and are cheap, fast and easy to obtain. The aim of this work was to evaluate the elemental (C, N) and molecular composition of the organic matter in relation to the grain-size distribution over surface sediments from Sepetiba Bay. Spatial distribution of grain-size was remarkable heterogeneous, with the internal area presenting the highest levels of fine sediments, organic carbon $(0.20-3.45 \%)$ and total nitrogen ( $<\mathrm{LQ}-0.36 \%$ ). Concentrations of organic carbon from surface sediments at the study area seem to be lower when compared to other degraded coastal systems. C/N ratio vary between 10 and 20 , suggesting a mix of allochthonous and autochthonous sources of organic matter to the sediments. DRIFT spectra presented prominent and marked bands assigned to organic and mineral functional groups bonding and the spectra identified chemical bonds of molecular composition such as aliphatic and aromatic functional groups.
\end{abstract}

Keywords: DRIFT; molecular composition; coastal zone.

\section{INTRODUÇÃO}

O melhor caminho para avaliar as condições ambientais de um ecossistema costeiro é através da análise dos seus sedimentos, uma vez que os poluentes tendem a se associar às partículas finas deste compartimento geoquímico. ${ }^{1,2}$ A matéria orgânica (MO), por afinidade química com oligoelementos e poluentes orgânicos, tende a se concentrar nas frações de argila e silte do sedimento. ${ }^{3}$ Portanto, o conhecimento sobre a composição química dos sedimentos é de grande relevância pela possibilidade de determinar a proveniência, a distribuição, a extensão, e também os possíveis riscos de contaminação. ${ }^{1,4,5}$

Os principais métodos para avaliação dos sedimentos envolvem a análise das suas características geoquímicas como granulometria, composição biogênica (carbono orgânico, nitrogênio e hidrogênio) e as concentrações de possíveis contaminantes. Os métodos convencionais recomendados para a avaliação desses parâmetros (e.g.: determinação de oligoelementos por fonte de plasma indutivamente acoplado - ICP), em geral, são procedimentos dispendiosos e/ou trabalhosos para a caracterização dos sedimentos. ${ }^{1,6}$ Por outro lado, técnicas espectroscópicas modernas são poderosas ferramentas para a investigação da camada superficial do sedimento. Essas técnicas apresentam vantagens sobre os outros métodos, pois são mais simples, baratas, exigem uma pequena quantidade de amostra, o tempo de análise é curto e o pré-tratamento da amostra é rápido e fácil. ${ }^{7-9}$

A análise espectroscópica de infravermelho com transformada de Fourier por refletância difusa (DRIFT) é um método clássico para a caracterização da estrutura molecular da matéria orgânica e inorgânica. ${ }^{10,11} \mathrm{O}$ princípio básico desta técnica é que as vibrações das ligações químicas absorvem energia infravermelha em números de onda específicos. ${ }^{12}$ Deste modo, as localizações espectrais da absorção de infravermelho são indicativas de ligações em grupos funcionais (e.g.: alifático, amídico, aromático e carboxílico) que podem fornecer informações importantes sobre a MO ${ }^{13-15}$ Portanto, a espectroscopia no infravermelho é um método amplamente utilizado para elucidar a estrutura molecular da matéria orgânica em sedimentos costeiros. . $^{2,10,11,16}$

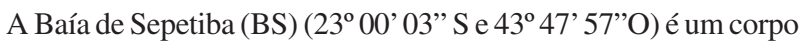
d'água semifechado com $447 \mathrm{~km}^{2}$, profundidade média de $6,0 \mathrm{~m}$ e com maré variando até $2,0 \mathrm{~m} \cdot{ }^{17} \mathrm{~A}$ bacia hidrográfica $\left(2000 \mathrm{~km}^{2}\right)$ é responsável pelo lançamento de aproximadamente 14 milhões de $\mathrm{m}^{3}$ dia $^{-1}$ de água doce na baía principalmente através do canal de São Francisco e rios da Guarda, Guandu e Cação. ${ }^{18}$

Nas últimas décadas, a BS sofreu um considerável desenvolvimento urbano (1,7 milhões de habitantes) e industrial. ${ }^{19,20} \mathrm{~A}$ bacia de drenagem da BS abriga terminais portuários e cerca de 400 indústrias, sendo a maioria delas metalúrgicas, o que contribui para o lançamento direto de resíduos ricos em metais e outras substâncias potencialmente tóxicas nos rios que abastecem as águas da baía. ${ }^{21,22} \mathrm{O}$ turismo também se apresenta como outro vetor de alteração antrópica na região, particularmente na porção leste (área interna) da Baía de Sepetiba. ${ }^{18}$

A contaminação do sedimento tem sido amplamente estudada na $\mathrm{BS}$, principalmente em relação à concentração de metais e muito pouco em relação aos contaminantes orgânicos. ${ }^{18,19,21,23-26}$ Poucos estudos abordam a influência da matéria orgânica na qualidade dos sedimentos nos últimos anos..$^{22,27}$

Estudos espectroscópicos na região do infravermelho para avaliar a matéria orgânica no sedimento da BS são inovadores. Portanto, o objetivo deste estudo foi caracterizar a composição da matéria orgânica nos sedimentos superficiais da Baía de Sepetiba através de análise elementar (carbono e nitrogênio) e molecular através da espectroscopia no infravermelho, avaliando a distribuição espacial destes em relação a granulometria. 


\section{PARTE EXPERIMENTAL}

\section{Amostragem e tratamento das amostras}

A amostragem do sedimento superficial $(0-10 \mathrm{~cm})$ foi realizada com busca-fundo de aço inox em 19 estações distribuídas em forma de arco ao longo da linha da costa da Baía de Sepetiba, priorizando o aporte da drenagem continental, tanto na área interna (região leste) da baía quanto na externa (região oeste - conexão com o mar) (Figura 1). Todas as amostras foram cuidadosamente transferidas, com espátula de aço inox para recipientes de vidro previamente descontaminados (Mufla $-450^{\circ} \mathrm{C}$ por $4 \mathrm{~h}$ ). Após a coleta as amostras foram mantidas resfriadas até serem armazenadas no laboratório, sob refrigeração $\left(-20^{\circ} \mathrm{C}\right)$, para posterior processamento das análises granulométricas, da composição elementar ( $\mathrm{C}$ e $\mathrm{N}$ ) e da análise espectroscópica da matéria orgânica.

Determinação da granulometria, carbono orgânico e nitrogênio total

Para a caracterização granulométrica, uma alíquota de $2,5 \mathrm{~g}$ de sedimento descarbonatado $\left(\mathrm{HCl} 0,1 \mathrm{~mol} \mathrm{~L}^{-1}\right)$ e seco $\left(\mathrm{a} 40^{\circ} \mathrm{C}\right.$, por $\left.72 \mathrm{~h}\right)$ foram colocadas em um tubo falcon de $50 \mathrm{~mL}$, e adicionados $40 \mathrm{~mL}$ de agente dispersante (hidróxido de sódio $40 \mathrm{~g} \mathrm{~L}^{-1}$ ), seguido de agitação por $24 \mathrm{~h}$. As frações granulométricas (grãos finos $\%<0,63 \mu \mathrm{m}$ e grãos grossos $\%>0,63 \mu \mathrm{m}$ ) foram determinadas pelo analisador de partículas a laser CILAS Shimadzu 1064 seguindo metodologia descrita na literatura. ${ }^{28}$ Os resultados foram obtidos com auxílio do programa GRADSTAT versão 8.

As análises dos teores de carbono orgânico $\left(\mathrm{C}_{\mathrm{org}}\right)$ e nitrogênio total $\left(\mathrm{N}_{\text {total }}\right)$ foram realizadas no LABCEN-UFSM (Laboratório de Pesquisa em Biotransformações de $\mathrm{C}$ e $\mathrm{N}$ ), a partir de alíquotas de $10 \mathrm{~g}$ de sedimento seco $\left(\mathrm{a} 40^{\circ} \mathrm{C}\right.$, por $72 \mathrm{~h}$ ), homogeneizado (gral e pistilo de ágata) e previamente descarbonatados $\left(\mathrm{HCl} 0,1 \mathrm{~mol} \mathrm{~L}^{-1}\right)$. As amostras foram analisadas em duplicata, utilizando o analisador elementar Flash EA1112 (Thermo Electron Corporation, Milan, Italy) seguindo metodologia descrita na literatura. ${ }^{29} \mathrm{O}$ limite de quantificação (LQ) do método foi de $0,01 \%$ w/w tanto para o carbono orgânico quanto para o nitrogênio total, calculado com base no ponto mais baixo da curva de calibração.

\section{Análise espectroscópica}

As amostras foram analisadas por espectroscopia de infravermelho com transformada de Fourier com refletância difusa (DRIFT). Previamente à análise, as amostras de sedimentos foram descarbonatadas e tratadas com solução de HF $10 \%$ (v/v) para remoção da fração mineral. Uma alíquota de $10 \mathrm{~g}$ de sedimento seco $\left(\mathrm{a} 40^{\circ} \mathrm{C}\right.$, por $72 \mathrm{~h}$ ) e descarbonatado ( $\left.\mathrm{HCl} 0,1 \mathrm{~mol} \mathrm{~L}^{-1}\right)$ foi colocada em um tubo falcon de $50 \mathrm{~mL}$, e adicionados $30 \mathrm{~mL}$ de $\mathrm{HF} 10 \%$, seguido de agitação mecânica por $2 \mathrm{~h}$, seguindo metodologia descrita na literatura. ${ }^{30}$ Após centrifugação (4.000 rpm, $10 \mathrm{~min}$ ) e separação do sobrenadante, o procedimento foi repetido 3 vezes até que o resíduo adquirisse coloração escura. A MO tratada com HF $10 \%\left(\mathrm{MO}_{\mathrm{HF}}\right)$ foi lavada cinco vezes com água deionizada, sendo 5,6 o pH do sobrenadante após a última lavagem. Em seguida, as amostras foram secas em estufa a $40{ }^{\circ} \mathrm{C}$ durante $48 \mathrm{~h}$.

Para averiguar se procedência do sinal emitido no infravermelho era de origem orgânica ou mineral (contra prova), selecionou-se duas amostras de $\mathrm{MO}_{\mathrm{HF}}$ (BS7 e BS19), as quais foram tratadas com $\mathrm{H}_{2} \mathrm{O}_{2}$ $30 \%$ até a amostra adquirir coloração clara, indicando remoção da MO.

Os espectros do DRIFT das amostras de $\mathrm{MO}_{\mathrm{HF}}$ foram obtidos na região espectral de $4000 \mathrm{~cm}^{-1}$ a $500 \mathrm{~cm}^{-1}$, com resolução de $4 \mathrm{~cm}^{-1}$, acúmulo de 32 scans e intensidade do sinal em absorbância, utilizando um Nicolet iS50 FT-IR (Thermo Fisher Scientific Corporation). Os espectros obtidos foram tratados utilizando-se o software Origin (versão 8) (OriginLab Corporation). Os espectros foram ajustados por uma linha de base em 3800, 1750, 980 e $500 \mathrm{~cm}^{-1}$ como pontos de absorbância zero. Em seguida os dados de intensidade e número de ondas das principais bandas do DRIFT foram identificados. A atribuição das bandas foi feita segundo dados disponíveis na literatura (Tabela 1). ${ }^{16,31-36}$

A partir dos dados de intensidade das bandas de absorção (após correção da linha de base), o grau de aromaticidade da $\mathrm{MO}_{\mathrm{HF}}$ foi determinado a partir do cálculo do índice de aromaticidade $\left(\mathrm{I}_{\mathrm{A}}\right)$. $\mathrm{O} \mathrm{I}_{\mathrm{A}}$ consiste na razão entre a intensidade da absorção em $1650 \mathrm{~cm}^{-1} \mathrm{e}$ em

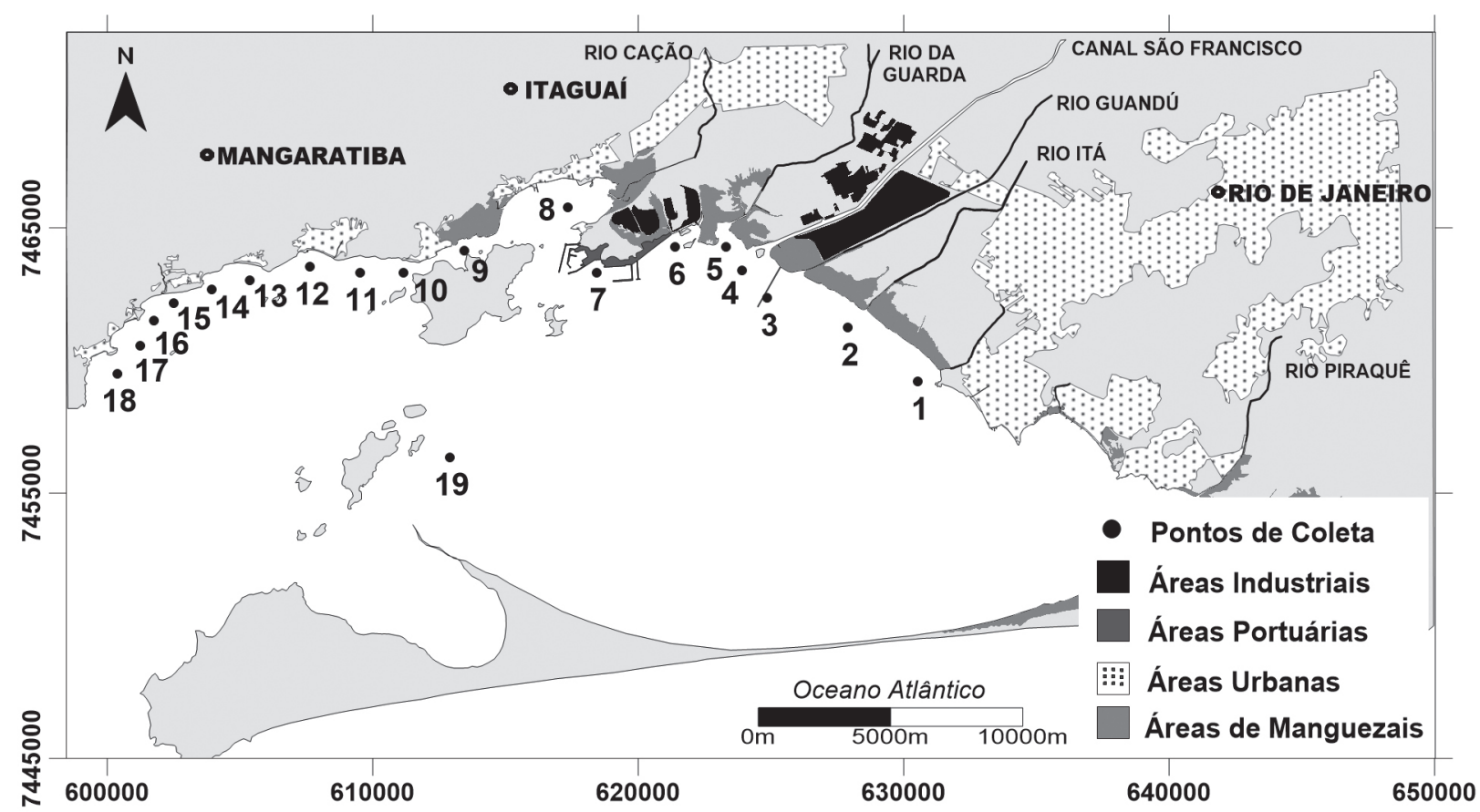

Figura 1. Pontos de amostragem e ocupação no entorno da Baía de Sepetiba-RJ 
Tabela 1. Principais bandas nos espectros de DRIFT e atribuições dos grupos funcionais orgânicos e minerais

\begin{tabular}{|c|c|}
\hline Número de onda $\left(\mathrm{cm}^{-1}\right)$ & Atribuição \\
\hline $3700-3500$ & Estiramento O-H de Al-OH e Si-OH \\
\hline $3400-3300$ & $\begin{array}{l}\text { Estiramentos O-H em interação com fenóis, } \\
\text { álcoois, carboxilas e amida }\end{array}$ \\
\hline $3100-2800$ & $\begin{array}{l}\text { Estiramento de } \mathrm{CH} \text { de grupos alifáticos }-\mathrm{CH}_{3} \\
\qquad\left(2925 \mathrm{~cm}^{-1}\right) \mathrm{e}-\mathrm{CH}_{2}\left(2850 \mathrm{~cm}^{-1}\right)\end{array}$ \\
\hline $1740-1680$ & $\begin{array}{c}\text { Estiramento } \mathrm{C}=\mathrm{O} \text { de carboxilas, Carbonilas e } \\
\text { Amida-I }\end{array}$ \\
\hline $1670-1610$ & $\begin{array}{l}\text { Estiramento } \mathrm{C}=\mathrm{C} \text { de grupos Aromáticos } \\
\qquad\left(1650 \mathrm{~cm}^{-1}\right)\end{array}$ \\
\hline $1570-1510$ & Estiramento $\mathrm{C}=\mathrm{N}$ e deformação $\mathrm{N}-\mathrm{H}$ de amidas \\
\hline $1170-568$ & $\begin{array}{l}\text { Estiramento C-O de carboidratos }\left(1050 \mathrm{~cm}^{-1}\right) \mathrm{e} \\
\text { Si-OH de silicatos e quartzo }\end{array}$ \\
\hline
\end{tabular}

$2925 \mathrm{~cm}^{-1}$ (aromático/alifáticos), conforme descrito na literatura. ${ }^{31,37}$ Esse procedimento para avaliação do grau aromaticidade, ou seja, o grau de saturação/condensação da matéria orgânica é reportado em outros estudos. ${ }^{33,38,39}$

\section{Análise estatística}

As análises estatísticas dos resultados de teor de finos, carbono orgânico, nitrogênio total e índice de aromaticidade foram realizadas com a utilização do programa STATISTICA 12.1 (Copyright $($ ) 1984-97, StatSoft, Inc.), onde a normalidade dos parâmetros foi testada por meio do teste Shapiro-Wilk. A maioria dos parâmetros não apresentou distribuição normal. Desta forma, empregou-se a correlação não paramétrica de Spearman, que opera com ranking das medidas para cada variável. ${ }^{40}$ Foram consideradas significativas as correlações e os testes cujo $\mathrm{p}<0,05$.

\section{RESULTADOS}

A distribuição granulométrica dos sedimentos superficiais da Baía de Sepetiba apresentou heterogeneidade espacial marcante entre as áreas interna (BS1 até BS7) e externa (BS8 até BS19). Os maiores teores de grãos finos $(<0,63 \mu \mathrm{m})$ foram encontrados nas estações da área interna $(0,88-26,09 \%)$, enquanto os menores teores foram encontrados nas estações da área externa (1,93-16,63\%) (Tabela 2).

As concentrações de carbono orgânico $(0,20-3,45 \%)$ seguiram o padrão de distribuição dos sedimentos finos (Tabela 2). As maiores concentrações de $\mathrm{C}_{\text {org }}(>1,5 \%)$ foram encontradas nas estações BS1, BS2, BS3, BS6, BS11 e BS18, todas com elevado teor de finos. Entretanto, teores de $\mathrm{C}_{\text {org }}(<0,5 \%)$ significativamente menores foram encontrados nas estações BS10, BS13, BS15, BS16, BS17. As concentrações de nitrogênio total ( $<\mathrm{LQ}-0,36 \%)$ também variaram de acordo com a distribuição dos sedimentos finos (Tabela 2). As maiores concentrações de $\mathrm{N}_{\text {total }}$ foram observadas nas estações BS1, BS2, BS3, BS6 e BS11, todas com elevador teor de finos. Em contrapartida, as demais estações apresentaram concentrações abaixo da média de $\mathrm{N}_{\text {total }}$. Ademais, o teor de finos e as concentrações de carbono orgânico e nitrogênio total apresentaram correlações positivas $(r=0,89 ; \mathrm{p}<0,05)$; um comportamento geoquímico clássico.

A razão molar $\mathrm{C} / \mathrm{N}$ para os sedimentos superficiais foi relativamente homogênea entre as áreas interna e externa da Baía de Sepetiba, variando, de forma geral, entre 10 e 20 (Tabela 2). Apenas as estações BS1, BS2 e BS8 apresentaram razão $\mathrm{C} / \mathrm{N}<10$. Por outro lado, as estações BS6, BS7, BS14, BS18 e BS19 apresentaram uma razão C/N $\geq 20$.

As amostras do DRIFT apresentaram o mesmo padrão de espectro (Figura 2). Apenas as estações BS1 e BS2 apresentaram comportamento espectral distinto do resto do grupo. Na região entre

Tabela 2. Teor de sedimentos finos $(<0,63 \mu \mathrm{m})$, concentrações de $\mathrm{C}_{\text {org }}$ e $\mathrm{N}_{\text {total }}$, razão elementar C/N e o $\mathrm{A}_{\mathrm{A}}$ nos sedimentos superficiais da Baía de Sepetiba

\begin{tabular}{|c|c|c|c|c|c|c|}
\hline Estações & & Teor de Finos (\%) & $\mathrm{N}_{\text {total }}(\%)$ & $\mathrm{C}_{\text {org }}(\%)$ & $\mathrm{C} / \mathrm{N}$ & $\mathrm{I}_{\mathrm{A}}$ \\
\hline BS1 & \multirow{7}{*}{ Área Interna } & 6,22 & 0,31 & 2,81 & 9,14 & $2,45 \pm 0,67$ \\
\hline BS2 & & 10,38 & 0,36 & 3,45 & 9,47 & $2,01 \pm 0,71$ \\
\hline BS3 & & 26,09 & 0,22 & 2,55 & 11,75 & $5,49 \pm 0,83$ \\
\hline BS4 & & 20,87 & 0,05 & 0,86 & 17,80 & $6,99 \pm 0,98$ \\
\hline BS5 & & 13,54 & 0,07 & 0,85 & 12,22 & $7,35 \pm 1,14$ \\
\hline BS6 & & 24,84 & 0,15 & 3,37 & 22,61 & $2,69 \pm 0,83$ \\
\hline BS7 & & 0,88 & $<\mathrm{LQ}$ & 0,80 & - & $6,65 \pm 1,21$ \\
\hline BS8 & \multirow{12}{*}{ Área Externa } & 6,47 & 0,06 & 0,61 & 9,98 & $7,26 \pm 1,11$ \\
\hline BS9 & & 9,36 & 0,07 & 0,88 & 13,32 & $2,19 \pm 0,59$ \\
\hline BS10 & & 6,90 & 0,02 & 0,29 & 13,74 & $6,96 \pm 0,90$ \\
\hline BS11 & & 16,63 & 0,14 & 1,45 & 10,03 & $5,41 \pm 0,96$ \\
\hline BS12 & & 15,92 & 0,09 & 1,00 & 11,13 & $6,78 \pm 1,14$ \\
\hline BS13 & & 4,33 & $<\mathrm{LQ}$ & 0,25 & - & $13,47 \pm 0,84$ \\
\hline BS14 & & 10,38 & 0,08 & 1,16 & 14,18 & $7,41 \pm 1,26$ \\
\hline BS15 & & 11,02 & 0,03 & 0,44 & 12,60 & $12,20 \pm 1,47$ \\
\hline BS16 & & 6,49 & 0,02 & 0,20 & 10,66 & $5,77 \pm 0,61$ \\
\hline BS17 & & 1,93 & $<\mathrm{LQ}$ & 0,22 & - & $18,73 \pm 1,24$ \\
\hline BS18 & & 9,78 & 0,06 & 2,64 & 42,19 & $7,04 \pm 0,16$ \\
\hline BS19 & & 8,99 & 0,09 & 1,07 & 11,92 & $2,99 \pm 0,19$ \\
\hline
\end{tabular}

LQ: limite de quantificação; $\mathrm{C}_{\text {org }}$ : carbono orgânico; $\mathrm{N}_{\text {total }}$ : nitrogênio total; $\mathrm{C} / \mathrm{N}$ razão carbono/nitrogênio; $\mathrm{I}_{\mathrm{A}}$ : índice de aromaticidade. 
$3600-3610 \mathrm{~cm}^{-1}$ foi identificado um ombro que pode ser atribuído aos estiramentos $\mathrm{O}-\mathrm{H}$ e Al-OH de silicatos. Já na região entre $3400-3280 \mathrm{~cm}^{-1}$ a banda larga é atribuída ao estiramento da ligação O-H e N-H em interação com grupos fenólicos, alcoólicos, carboxílicos e amida. ${ }^{16}$ As bandas 2925 e $2850 \mathrm{~cm}^{-1}$ são atribuídas aos estiramento das ligações $\mathrm{C}-\mathrm{H}$ em grupos alifáticos. ${ }^{16,31}$

A área entre $1800 \mathrm{~cm}^{-1}$ e $900 \mathrm{~cm}^{-1}$, corresponde à região denominada "impressão digital da MO" (finger print). ${ }^{16,31}$ Nos espectros das estações BS1 e BS2 observou-se intensas bandas sobrepostas nesta área $\left(1800-900 \mathrm{~cm}^{-1}\right)$. A banda entre $1700-1720 \mathrm{~cm}^{-1}$ pode ser atribuída às vibrações de estiramento das ligações $\mathrm{C}=\mathrm{O}$ em grupos carboxílicos..$^{32} \mathrm{~A}$ banda intensa, em $1650 \mathrm{~cm}^{-1}$, pode ser atribuída às vibrações de estiramento das ligações $\mathrm{C}=\mathrm{C}$ presente em compostos aromáticos, ${ }^{33}$ e absorções na faixa de $1560-1548 \mathrm{~cm}^{-1}$ podem ser atribuídas às vibrações de estiramento simétrico das ligações $\mathrm{C}=\mathrm{N}$ em amida-II $\left(1560 \mathrm{~cm}^{-1}\right){ }^{34}$

A banda de absorção em $1375 \mathrm{~cm}^{-1}$ pode ser associada às vibrações de estiramento da ligação $\mathrm{C}-\mathrm{O}$ e deformação da ligação
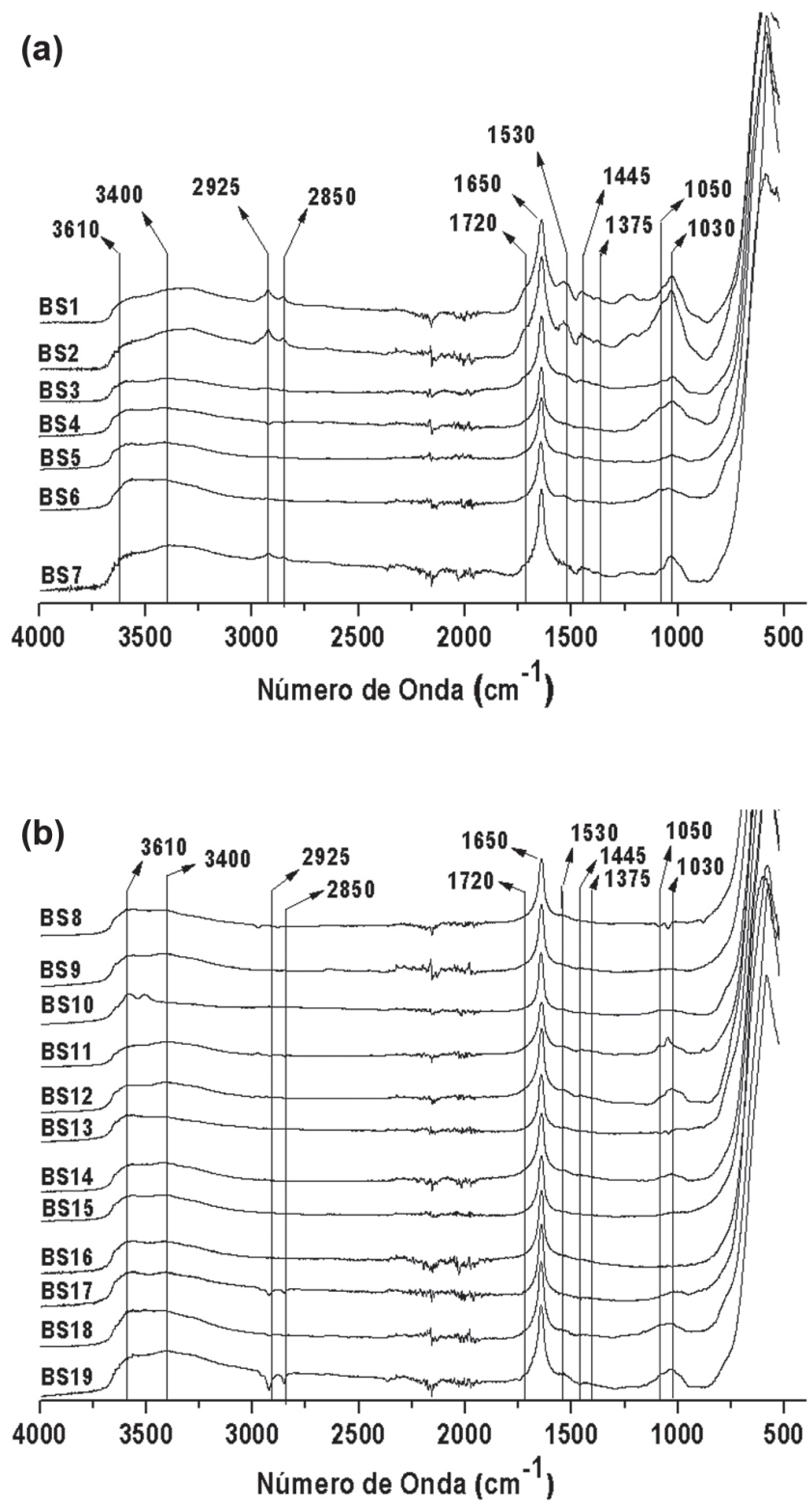

Figura 2. Espectro do DRIFT da MO ${ }_{H F}$ para as estações da área interna (a), e da área externa (b) da Baía de Sepetiba, mostrando as bandas de absorção características dos grupos funcionais orgânicos e minerais
O-H em grupos fenóis (1380-1360 $\left.\mathrm{cm}^{-1}\right) \cdot{ }^{35}$ Absorções na faixa de $1170-568 \mathrm{~cm}^{-1}$ podem ser atribuídas ao estiramento das ligações $\mathrm{C}-\mathrm{O},-\mathrm{COH}$ e C-O-C em carboidratos $\left(1050 \mathrm{~cm}^{-1}\right)$ e estiramento das ligações Al-O-Si, Si-O-Si e Si-O em minerais de argila e quartzo (entre 1170 e $\left.568 \mathrm{~cm}^{-1}\right){ }^{36}$

A faixa de absorção intensa em torno de $650 \mathrm{~cm}^{-1}$ é provavelmente atribuída à absorção de minerais recém-formados $\left(\mathrm{CaF}_{2}\right)$, durante o tratamento com $\mathrm{HF} 10 \%$, para isolar a $\mathrm{MO}$ e/ou minerais residuais. ${ }^{41,42}$ A alta intensidade desta banda atesta a presença de uma quantidade significativa de minerais neoformados e/ou residuais durante a desmineralização. ${ }^{16}$

Nos espectros das amostras BS7 e BS19, após tratamento com peróxido de hidrogênio, as bandas da região em torno de $3400 \mathrm{~cm}^{-1}$ e entre 1750 e $1400 \mathrm{~cm}^{-1}$ diminuíram drasticamente ou, até mesmo, desapareceram, confirmando que as atribuições feitas nessa região espectral pertencem a grupos orgânicos (Figura 3). As bandas na faixa apresentadas entre $1170-568 \mathrm{~cm}^{-1}$ podem ser atribuídas às frações minerais remanescentes que, após a eliminação da MO, se intensificaram.

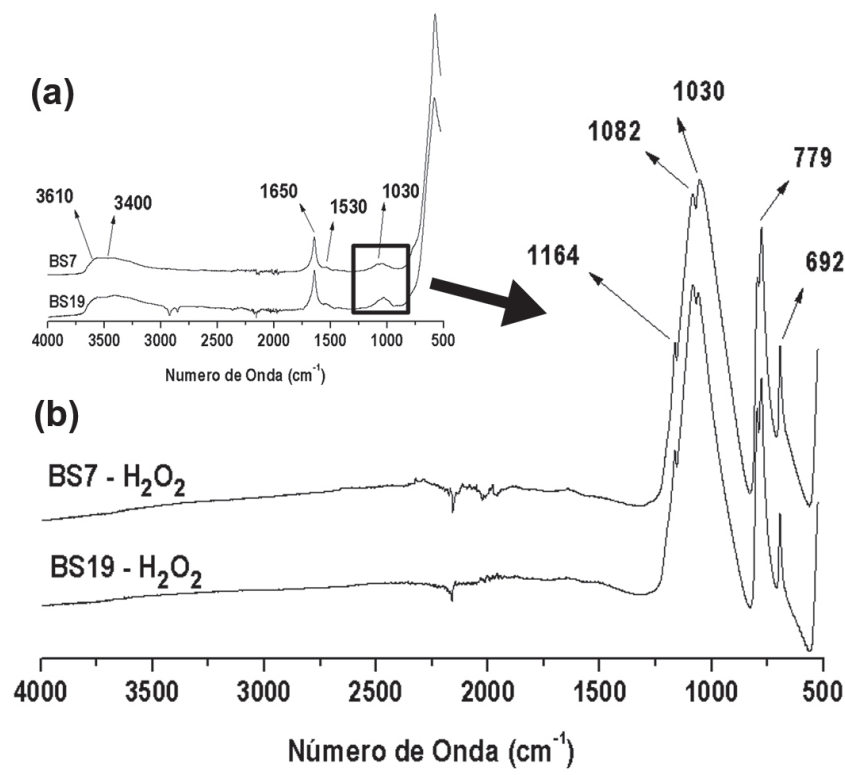

Figura 3. Espectro do DRIFT da $\mathrm{MO}_{H F}$ das amostras BS7 e BS19 (a) e das amostras posteriormente tratadas com $\mathrm{H}_{2} \mathrm{O}_{2}(b)$

O índice de aromaticidade $\left(\mathrm{I}_{\mathrm{A}}\right)$ variou entre 2,01 e 18,73 (Tabela 2). As amostras de sedimento superficial com menor grau de aromaticidade $(<3,00)$ foram encontradas nas estações da área interna, as estações BS1, BS2, BS3 e BS6. Por outro lado, os sedimentos das estações da área externa apresentaram alto grau de aromaticidade $(>7,00)$. Os valores de $\mathrm{I}_{\mathrm{A}}$ se correlacionaram positivamente com $\mathrm{C} / \mathrm{N}$ $(\mathrm{r}=0,42 ; \mathrm{p}<0,10)$.

\section{DISCUSSÃO}

A distribuição granulométrica dos sedimentos superficiais da BS é resultado das correntes horárias e da distância da entrada da baía, onde a ação das correntes de maré e das ondas são mais intensas. ${ }^{43,44}$ Desta forma, os sedimentos da área externa apresentam granulometria arenosa e baixas concentrações de carbono e nitrogênio. Por outro lado, a medida em que ocorre a redução da ação hidrodinâmica em direção a parte interna da baía, os teores de sedimentos finos e a concentração de matéria biogênica tendem a aumentar. A matéria orgânica sedimentar está fortemente relacionada ao conteúdo de grãos finos $(<0,63 \mathrm{~mm}) .{ }^{45}$ Além disso, existe a presença de extensos 
manguezais nesta região, considerados como fontes significativas de matéria orgânica. ${ }^{46}$ Os resultados encontrados no presente estudo apresentam a mesma variação de concentração e distribuição espacial de granulometria e carbono orgânico observado anteriormente na Baía de Sepetiba. ${ }^{20}$

A concentração de $C_{\text {org }}(0,20-3,45 \%)$ na $\mathrm{BS}$ é relativamente baixa quando comparada a teores de carbono orgânico em sedimentos lamosos de sistemas costeiros altamente degradados como a Baía de Guanabara, no Rio de Janeiro $(0,03-5,15 \%)$ ou a Baía de Santos, em São Paulo $(0,09-5,78 \%))^{47,48} \mathrm{O}$ menor teor de carbono orgânico na Baía de Sepetiba pode ser atribuído à predominância de sedimentos arenosos. Contudo, a variação da concentração de carbono observada nos estudos de Figueiredo et al. ${ }^{19}$ (2008) $(0,40-1,84 \%)$, Carreira et al. ${ }^{18}$ (2009) $(0,66-2,62 \%)$, Ribeiro et al. ${ }^{20}$ (2013) (2,00-3,00\%) e no presente estudo indicam um aumento no teor de matéria orgânica na Baía de Sepetiba. Provavelmente, as contribuições significativas das cargas orgânicas provenientes dos manguezais e do aporte fluvial, devem ser as responsáveis pelo contínuo enriquecimento de MO nos sedimentos. O Rio Guandu e o canal do São Francisco contribuem com $96 \%$ do material particulado que entra na baía e podem representar uma fonte significativa de material orgânico derivado de solos continentais e da vegetação terrestre, além disso, as florestas de manguezais podem produzir um enriquecimento localizado de matéria orgânica dissolvida. ${ }^{49}$

A alta correlação entre $\mathrm{C}_{\text {org }}$ e $\mathrm{N}_{\text {total }}$ é um indício de que as fontes de matéria orgânica estão presentes em proporções constantes ao longo das estações estudadas..$^{50}$ Considerando a eficiência da hidrodinâmica local em transportar os sedimentos finos de uma área para outra, a proporção entre carbono e nitrogênio pode ser atribuída a uma eficiente mistura entre fontes terrestre e marinha antes da deposição final. ${ }^{51}$

A razão $\mathrm{C} / \mathrm{N}$ obtida para a maioria dos sedimentos superficiais analisados indicam que a matéria orgânica presente no sedimento pode ser uma mistura entre material autóctone e alóctone $(10<\mathrm{C} / \mathrm{N}<20){ }^{52}$ Como já indicado acima, a hidrodinâmica apresenta eficiência na mistura de materiais de diversas fontes. As estações BS6 e BS18 apresentaram razão $\mathrm{C} / \mathrm{N}$ indicativas de insumos alóctones elevados (C:N $\geq 20) .{ }^{53,54}$ Segundo Carreira et al. (2009) a principal fonte de matéria orgânica para Baía de Sepetiba é de origem alóctone (florestas de manguezais). Apenas as estações BS1, BS2 e BS8 apresentaram razão $\mathrm{C} / \mathrm{N}$ com predominância de matéria orgânica de origem autóctone (fitoplâncton; $\mathrm{C}: \mathrm{N}<10) .{ }^{53}$ Contudo, considerando que essas estações estão próximas a foz de rios contaminados por efluentes domésticos, ${ }^{55}$ a adsorção de nitrogênio inorgânico dissolvido, principalmente amônia, às partículas finas podem estar mascarando a razão C/N. ${ }^{56}$

Os espectros do DRIFT para a $\mathrm{MO}_{\mathrm{HF}}$ são similares aos espectros de matéria orgânica em sedimentos costeiros reportados na literatura. ${ }^{2,10,11,16}$ A razão $\mathrm{C} / \mathrm{N}$ sugere que a composição molecular da $\mathrm{MO}_{\mathrm{HF}}$ é derivada da mistura de materiais provenientes de fontes terrestre e marinha. A realização da contraprova determinou que as bandas de absorção presentes na faixa do $3600-1360 \mathrm{~cm}^{-1}$ são provenientes das ligações de grupos funcionais orgânicos, uma vez que estes desapareceram após a remoção da $\mathrm{MO}$ com $\mathrm{H}_{2} \mathrm{O}_{2}$.

A presença de ligações de grupos carboxílicos pode sugerir a ocorrência do processo de carboxilação durante a degradação de hidrocarbonetos policíclicos aromáticos (HPA) por bactérias no sedimento. ${ }^{57}$ Estudos prévios identificaram compostos aromáticos em sedimentos da Baía de Sepetiba. ${ }^{23}$ A presença de fortes bandas de absorção em ligações de grupos carboxílico, e bandas menos proeminentes na faixa do $1610-1620 \mathrm{~cm}^{-1}$ (ligações do tipo $\mathrm{C}=\mathrm{C}$ e COO-) sugerem a presença de uma matéria orgânica de origem vascular altamente processada, ${ }^{58}$ reafirmando a contribuição das florestas de manguezais como principais fontes de MO para BS. A presença de bandas de absorção entre $2925-2850 \mathrm{~cm}^{-1}$ podem se referir aos grupos metil $\left(-\mathrm{CH}_{3}\right)$ e metileno $\left(-\mathrm{CH}_{2}\right)$ que são característicos de material húmico. ${ }^{32}$ As substâncias húmicas são os materiais orgânicos mais difundidos e ubíquos em ambientes aquáticos e terrestres..$^{59}$ Por fim, a presença de bandas de grupos carbóxilos e alifáticos, assim como ligações de compostos nitrogenados, sugerem a presença de detritos de algas verdes. ${ }^{60}$

$\mathrm{O} \mathrm{I}_{\mathrm{A}}$ obtido sugere que a matéria orgânica sedimentar da área interna da BS apresenta valores de aromaticidade menores quando comparados a área externa. Os resultados dos espectros corroboram esse padrão, pois na área interna a intensidade do sinal de compostos alifáticos $(\mathrm{C}-\mathrm{H})$ foi maior do que o encontrado na área externa. Este comportamento oposto pode estar relacionado a degradação (química e biológica) dos compostos alifáticos presentes na MO da área externa, diminuindo assim, a intensidade do sinal de alifáticos nesta região. ${ }^{31}$

A hidrodinâmica e o aporte fluvial de MO também são peças fundamentais para a distinção nos valores de aromaticidade entre as áreas interna e externa da BS. A circulação e a renovação da água são mais intensas na área externa e ${ }^{43,44}$ portanto, favorecem a degradação química (oxidação) e biológica (processos aeróbios são mais eficientes que os anaeróbios) de compostos mais simples $(\mathrm{C}-\mathrm{H})$, ou carreamento da $\mathrm{MO} .{ }^{47}$ Por outro lado, a circulação na área interna é menos intensa e favorece o acúmulo e a preservação de MO drenada para BS, contribuindo provavelmente para o incremento de compostos alifáticos. A MO acumulada nesta região apresenta uma variedade de compostos orgânicos, como mostram os espectros (Figura 2), e este acúmulo implicaria na redução das condições óxicas, retardando a degradação biológica.

A correlação positiva entre o $\mathrm{I}_{\mathrm{A}}$ e a razão $\mathrm{C} / \mathrm{N}$ é um indício de que a aromaticidade aumenta quando a razão elementar indica origem terrestre, ou seja, maior grau de recalcitrância quando comparado com a origem marinha. ${ }^{53}$ Desta forma, o índice de aromaticidade pode ser uma ferramenta útil para o estudo de matéria orgânica sedimentar indicando o seu grau de recalcitrância.

As atividades portuárias e uma série de insumos urbanos, industriais e atmosféricos são fontes de compostos aromáticos para a zona costeira, cuja presença de compostos de origem petrogênica e pirolítica e risco ambiental, já foram confirmadas por trabalhos pretéritos na Baía de Sepetiba. ${ }^{19,23}$ Mais ainda, as menores concentrações de compostos alifáticos em relação a aromáticos na Baía de Sepetiba também foram descritas na literatura. ${ }^{23}$

\section{CONCLUSÃO}

A composição granulométrica dos sedimentos superficiais da Baía de Sepetiba é resultado das condições hidrodinâmicas locais que favorecem a dispersão dos sedimentos finos na área externa e o acúmulo deste material na área interna do estuário. Em função da distribuição de sedimentos finos, as maiores concentrações de carbono e nitrogênio foram encontradas na área interna, e as menores concentrações na área externa da Baía de Sepetiba. A hidrodinâmica também parece ser responsável por manter as proporções constantes de $\mathrm{C}_{\text {org }}$ e $\mathrm{N}_{\text {total }}$ ao longo das estações de coleta.

As concentrações de carbono orgânico encontradas no sedimento superficial da BS, ainda que sejam inferiores quando comparadas aos teores encontrados em sistemas costeiros degradados, indicam que o acúmulo de $\mathrm{C}_{\text {org }}$ tem aumentado nos últimos anos. As razões $\mathrm{C} / \mathrm{N}$ indicam que a principal contribuição de matéria orgânica no sedimento da BS é uma mistura de origem autóctone e alóctone.

Os espectros do DRIFT fornecem informações importantes a respeito da estrutura molecular (grupos funcionais orgânicos e minerais), composição e transformações da matéria orgânica, além de ser uma técnica mais rápida e menos onerosa quando comparada com as análises cromatográficas. A matéria orgânica dos sedimentos 
superficiais apresentou um alto grau de aromaticidade na área externa em comparação com a área interna, possivelmente em decorrência do intenso processo de degradação dessa área que resulta no decréscimo nos níveis de alifáticos. A correlação significativa entre a razão $\mathrm{C} / \mathrm{N}$ e o $\mathrm{I}_{\mathrm{A}}$ endossa o DRIFT como uma ferramenta útil e confiável para a análise preliminar do grau de aromaticidade/recalcitrância da matéria orgânica sedimentar.

\section{AGRADECIMENTOS}

A Coordenação de Aperfeiçoamento de Pessoal de Nível Superior (CAPES) pelo suporte financeiro e pela concessão de bolsas de pesquisa (doutorado e mestrado) para A. Carvalho; L. Silva; V. Moreira; M. Vicente e A. Andrade.

\section{REFERÊNCIAS}

1. Moros, J.; Cassella, R. J.; Barciela-Alonso, M. C.; Vib. Spectrosc. 2010, 53, 204.

2. Oudghiri, F.; García-Morales J. L.; Rodríguez-Barroso, M. R.; Water, Air, Soil Pollut. 2014, 225, 1.

3. Sterckeman, T.; Douay, F.; Baize, D.; Fourrier, H.; Proix, N.; Schvartz, C.; Geoderma 2006, 136, 912.

4. Li, X.; Wai, O. W. H.; Li, Y. S.; Coles, B. J.; Ramsey, M. H.; Thornton, I.; Appl. Geochem. 2000, 15, 567.

5. Turner A.; Estuarine, Coastal Shelf Sci. 2000, 50, 355.

6. Moros, J.; Barciela-Alonso, M. C.; Pazos-Capeáns, P.; Anal. Chim. Acta 2008, 624, 113.

7. Poulenard, J.; Perrette, Y.; Fanget, B.; Quetin, P.; Trevisan, D.; Dorioz, J. M.; Sci. Total Environ. 2009, 407, 2808

8. Janik, L. J.; Skjemstad, J. O.; Shepherd, K. D.; Spouncer, L. R.; Aust. J. Soil Res. 2007, 45, 73 .

9. Viscarra-Rossel, R. A.; Walvoort, D. J. J.; McBratney, A. B.; Janik, L. J.; Skjemstad, J. O.; Geoderma 2006, 131, 59.

10. Ibrahim, M.; Hameed, A. J.; Jalbout, A.; Appl. Spectrosc. 2008, 62, 306.

11. Abdel-Gawad, F. K.; Ibrahim, H. S.; Ammar, N. S.; Ibrahim, M.; Spectrochim. Acta, Part A 2012, 97, 771.

12. Veerasingam, S.; Venkatachalapathy, R.; Infrared Phys. Technol. 2014, 66, 136.

13. Viscarra-Rossel, R. A.; McBratney, A. B.; Jeon, Y. S.; Odeh, I. O. A.; Aust. J. Soil Res. 2008, 46, 1.

14. Calderón, F. J.; Reeves, J. B.; Collins, H. P.; Paul, E. A.; Soil Sci. Soc. Am. J. 2011, 75, 568.

15. Tatzber, M.; Mutsch, F.; Mentler, A.; Geoderma 2011, 166, 162.

16. Hatcher, P. G.; Ravin, A.; Behar, F.; Baudin, F.; Org. Geochem. 2014, 75,8 .

17. Lacerda, L. D.; Gonçalves, G. O.; Mar. Chem. 2001, 76, 47.

18. Carreira, R. S.; Ribeiro, P. V.; Silva, C. E. M.; Farias, C. O.; Quim. Nova 2009, 32, 1805.

19. Figueiredo, L. H. M.; Wagener, A. D. L. R.; Dagautc, J.; Saliot, A.; J. Braz. Chem. Soc. 2008, 19, 516.

20. Ribeiro, A. P.; Figueiredo, A. M. G.; Santos, J. O.; Mar. Pollut. Bull. 2013, 68, 55 .

21. Paraquetti, H. H. M.; Lacerda, L. D.; Almeida, M. D.; Marins, R. V.; Mounierc, S.; J. Braz. Chem. Soc. 2007, 18, 1259.

22. Rezende, C. E.; Lacerda, L. D.; Ovalle, A. R. C.; Silva, L. F. F.; Braz. J. Biol. 2007, 67, 673.

23. Mounier, S.; Lacerda, L. D.; Marins, R. V.; Bemaim, J.; Bull. Environ. Contam. Toxicol. 2001, 67, 519.

24. Lacerda, L. D.; Marins, R. V.; Paraquetti, H. H. M.; Mounier, S.; Benaim, J.; Fevrier, D.; J. Braz. Chem. Soc. 2001, 12, 93.

25. Lacerda, L.; Molisani, M.; Mar. Pollut. Bull. 2006, 52, 969.
26. Pinheiro, P. P. O.; Massone, C. G.; Carreira, R. S.; Mar. Pollut. Bull. 2017, 120, 6 .

27. Xavier, L.; Mounier, S.; Paraquetti, H. H. M.; Mar. Environ. Res. 2008, 65,148

28. Hedges, J. I.; Stern, J. H.; Limnol. Oceanogr. 1984, 29, 657.

29. EMBRAPA; Manual de Métodos de Análise de Solo, $1^{\mathrm{a}}$ ed.; EMBRAPASNLCS: Rio de Janeiro, 1997.

30. Gonçalves, C. N.; Dalmolin, R. S. D.; Dick, D. P.; Knicker, H.; Klamt, E.; Kögel-Knabner, I.; Geoderma 2003, 116, 373.

31. Chefetz, B.; Hatcher, P. G.; Hadar, Y.; Chen, Y.; J. Environ. Qual. 1996, 25,776 .

32. Stevenson, F. J.; Humus Chemistry. Genesis, Composition, Reactions, $2^{\text {nd }}$ ed.; Wiley: New York, 1994

33. Inbar, Y.; Chen, Y.; Hadar, Y.; Soil Sci. Soc. Am. J. 1989, 53, 1695.

34. Haberhauer, G.; Gerzabek, M. H.; Vib. Spectrosc. 1999, 19, 413.

35. Bornemann, L.; Welp, G.; Amelung, W.; Soil Sci. Soc. Am. J. 2010, 74, 1147.

36. Pirie, A.; Singh, B.; Islam, K.; Aust. J. Soil Res. 2005, 43, 713.

37. Dick, D. P.; Knicker, H.; Ávila, L. G.; Inda, A. V.; Giasson, E.; Bissani, C. A.; Org. Geochem. 2006, 37, 1537.

38. Dick, D. P.; Silva, L. D.; Inda, A. V.; Knicker, H.; Rev. Bras. Cienc. Solo 2008, 32, 2289.

39. Dick, D. P.; Gomes, J.; Bayer, C.; Bodmann, B.; Rev. Bras. Cienc. Solo 2000, 24, 285.

40. Zar, J. H.; Biostatistical Analysis, $2^{\text {nd }}$ ed.; Prentice-Hall Inc.: New Jersey, 1982.

41. Garcette-Lepecq, A.; Derenne, S.; Largeau, C.; Bouloubassi, I.; Saliot, A.; Org. Geochem. 2000, 31, 1663.

42. Riboulleau, A.; Derenne, S.; Sarret, G.; Largeau, C.; Baudin, F.; Connan, J.; Org. Geochem. 2000, 31, 1641.

43. Signorini, S. R.; Brazilian Journal of Oceanography 1980, 29, 57.

44. Molisani, M. M.; Kjerfve, B.; Silva, A. P.; Lacerda, L. D.; J. Hydrol. 2006, 331, 425 .

45. Burdige, D. J.; Chem. Rev. (Washington, DC, U. S.) 2007, 107, 467.

46. Neves, L. M.; Pereira, H. H.; Costa, M. R.; Araújo, F. G.; Revista Brasileira de Zoologia 2006, 23, 421.

47. Sabadini-Santos, E.; Silva, T. S.; Lopes-Rosa, T. D.; Mendonça-Filho, J. G.; Santelli, R. E.; Crapez, M. A. C.; Water, Air, Soil Pollut. 2014, 225, 1949.

48. Siqueira, G. W.; Braga, E. S.; Mahíques, M.; Aprile, F.; Arq. Cienc. Mar. 2006, 39, 18.

49. Rezende, C. E.; Pfeiffer, W. C.; Martinelli, L. A.; Tsamakis, E.; Hedges, J. I.; Keil R. G.; Estuarine, Coastal Shelf Sci. 2010, 87, 479.

50. Ruttenberg, K. C.; Goñi, M. A.; Mar. Geol. 1997, 139, 123.

51. Sabadini-Santos, E.; Jennerjahn, T.; Medeiros, P. R. P.; Souza, W. F. L.; Knoppers, B. A.; Geochim. Bras. 2013, 27, 37.

52. Stein, R. Em: Lecture Notes in Earth Sciences, $1^{\text {st }}$ ed.; Springer-Verlag: Berlin, 1991.

53. Meyers, P. A.; Chem. Geol. 1994, 114, 289

54. Bordovskiy, O. K.; Mar. Geol. 1965, 3, 33.

55. Coimbra, A.; Dissertação de Mestrado, Universidade Federal Fluminense, Brasil, 2003.

56. Hedges, J. I.; Clark, W. A.; Quay, P. D.; Richey, J. E.; Devol, A. H.; Santos, M.; Limnol. Oceanogr. 1986, 31, 717.

57. Schmitt, J.; Nivens, D.; White, D. C.; Flemming, H. C.; Water Sci. Technol. 1995, 32, 149.

58. Baes, A. U.; Bloom, P. R.; Soil Sci. Soc. Am. J. 1989, 53, 695.

59. Parlanti, E.; Sierra, M. M. D.; Giovanela, M.; Soriano-Sierra, E. J.; Geochem. J. 2011, 38, 255.

60. Lartiges, B. S.; Deneux-Mustin, S.; Villemin, G.; Water Res. 2001, 35, 808 . 